\title{
Current aspects of $\mathrm{ABO}$-incompatible liver transplantation
}

\author{
Naoki Kawagishi ${ }^{1}$ and Susumu Satomi ${ }^{2}$ \\ ${ }^{1}$ Division of Transplantation, Reconstruction, and Endoscopic Surgery, Tohoku University Hospital, 1-1 Seiryou-machi, Aoba-ku, Sendai 980-8574, Japan \\ ${ }^{2}$ Tohoku University, 2-1-1Katahira, Aoba-ku, Sendai, Japan
}

\begin{abstract}
Liver transplantation is well recognized as treatment for patients with end-stage liver disease. Although the source of donors is limited, ABO blood type matched transplantation is commonly performed in deceased donor liver transplantation. On the other hand, where deceased donors are rarely available, a graft obtained from a family member of the recipient is mainly employed and thus ABO-incompatible (ABO-I) living donor liver transplantation (LDLT) becomes unavoidable. This review article describes the history and current aspects of ABO-I liver transplantation including our own Japanese experience. The main target of overcoming the ABO incompatibility is prevention, diagnosis, and treatment of antibody mediated rejection (AMR). To prevent AMR, plasma exchange (PE) was historically performed followed by local infusion therapy of rituximab, and intravenous immunoglobulin infusion (IVIG). To establish the diagnosis, histopathological findings of biopsy specimens are crucial, but peripheral serum antibody titers for the ABO blood type are helpful. Conventional anti-rejection rescue therapy such as steroid bolus is not enough to treat AMR, thus PE and rituximab should be added. Nevertheless, once AMR is triggered, most of the recipients will end up with graft necrosis within three weeks following transplantation. In recent years, owing to advances in the prevention and treatment of AMR after ABO-I therapeutic interventions, short- and even long-term survival of the ABO-I LT is guaranteed. However, ABO-I LT still presents with multiple severe complications that require further research in terms of immune modulation and control of infection.
\end{abstract}

\section{Introduction}

Blood-group type matching has been well known as the principal rule in organ transplantation. However, as the possibility of obtaining grafts from living donors is very low, adjustment of blood type is sometimes very difficult. On the other hand, and particularly in Far East countries, it is even harder to obtain grafts from deceased donors; thus, living donors must be used for the rescue of patients with endstage liver disease. In Japan, this background has led to an increase of ABO-Incompatible (ABO-I) living donor transplantation, especially in the field of kidney transplantation. Although various therapeutic modalities emerged from the experience with renal transplantation, further adjusting strategies are required for the success of ABO-I liver transplantation [1]. Moreover, the hurdle posed by ABO-I liver transplantation is higher than that in kidney transplantation. This review describes the current advances of ABO-I liver transplantation including the achievements fruit of our predecessors' efforts, and a proposal to improve outcomes and reduce $\mathrm{ABO}$ incompatibility related complications in liver transplantation.

\section{Milestones of ABO-incompatible liver transplantation}

Although $\mathrm{ABO}$ incompatible renal and liver transplants have been widely performed in Japan, the number of patients benefitting by them gradually and steadily increased after the developments reported by Alexandre et al. in 1985 [2]. Particularly, in the field of liver transplantation, survival of recipients was poor in the early times, and the procedure was performed in few selected cases. In emergency situations, when given no choice but to proceed using the first available organ, ABO-I liver transplantation was not considered as a contraindication. In the 1980's Gordon et al. [3] reviewed 671 first, second, and third orthotopic liver allografts in 520 patients to determine the effect of donor-recipient mismatch or incompatibility for ABO blood group on graft survival. They found a significant advantage for $\mathrm{ABO}$ donor-recipient identity, especially in adults and for first grafts. However, a surprisingly large number of $\mathrm{ABO}$ incompatible grafts had been successful. So they recommended that non-identical or incompatible grafts be limited to patients such as small children for whom the supply of available donors is severely limited or for patients in urgent need of transplantation or retransplantation [3]. In the late 1980s', Gugenhein et al. [4] found in a study of 234 liver transplants that the 2-year graft survival for ABO-compatible elective (80\%) or emergency (76\%) liver transplants was significantly higher than that for ABO-incompatible emergency liver transplants (30\%). The low survival of $\mathrm{ABO}$-incompatible liver allografts was therefore not related to the emergency conditions. Among 17 patients who received ABOincompatible liver allografts, six developed primary antibody mediated rejection, with hemorrhagic infiltration of portal tracts and deposition of IgM and fibrinogen on sinusoidal and endothelial cells. Other disadvantages of $\mathrm{ABO}$-incompatible liver allografts were significantly higher frequencies of severe rejection crises, arterial thrombosis, and cholangitis. However, the 1-year survival rate of the patients who received $\mathrm{ABO}$-incompatible grafts was $66 \%$, so the use of $\mathrm{ABO}$ incompatible liver grafts is justifiable in emergencies, when no other donor is available; such transplants may help to save the patient, even at the cost of retransplantation in half of the cases [4].

Correspondence to: Naoki Kawagishi, Division of Transplantation, Reconstruction, and Endoscopic Surgery, Tohoku University Hospital, 1-1 Seiryou-machi, Aoba-ku, Sendai 980-8574, Japan, Tel: +81-22-717-7214; Fax: +81-22-717-7217; E-mail: kawan@med.tohoku.ac.jp

Key words: liver transplantation, ABO-incompatible, plasma exchange, infusion therapy, living donor, rituximab, antibody mediated rejection

Received: February 05, 2016; Accepted: March 05, 2016; Published: March 08, 2016 
The turning point of ABO-I living donor liver transplantation (ABO-I LDLT) was in the 2000's when Tanabe et al. reported the case of two adult patients who had undergone ABO-ILDLT [5]. The antirejection therapy included multiple perioperative plasmapheresis, splenectomy, systemic triple immunosuppressive regimen with tacrolimus, methylprednisolone, and cyclophophamide or azathioprine. In addition to these conventional approaches, they performed intraportal infusion therapy with methylprednisolone, prostaglandin E1, and gabexate mesilate after transplantation. With their protocol, antidonor blood group antibody titers remained low without any evidence of rejection or vascular complications throughout the postoperative course in both patients. Besides, biliary complications were transient and resolved completely. The second innovation was introduced by a Kyoto group of specialists [6]. The immunosuppressive therapy included multiple perioperative plasmapheresis, splenectomy, and treatment with tacrolimus, methylprednisolone, and cyclophosphamide. The dose and blood level of tacrolimus were the same as in ABO-compatible cases. In addition to these therapies, they introduced the administration of intrahepatic arterial infusion of prostaglandin (PG) E1 alone to the armamentarium. After perioperative plasmapheresis and administration of cyclophosphamide, antidonor blood group antibody titers remained undiluted and there were no vascular complications throughout the postoperative course. The authors concluded that intrahepatic artery infusion of PGE1 was useful in adult ABO-I liver transplantation. The third innovation was use of rituximab. This new strategy for ABO-I LDLT has been adopted based on the experience with rituximab in kidney transplant recipients [7]. Rituximab is a monoclonal chimeric human-murine anti-CD20 antibody that depletes $\mathrm{B}$ cells by complement-dependent cytotoxicity, drug-induced apoptotic death, and antibody-dependent cellular cytotoxicity. Rituximab has been approved for the treatment of relapsed or refractory B-cell nonHodgkin's lymphoma. Monteiro et al. reported the first case of rituximab use in a 15-year-old boy subjected to emergency ABO-I deceased donor liver transplantation [8]. We first use rituximab in LDLT recipients in 2002 [9], and reported the successful treatment of rejection episodes in a patient who had not responded to other forms of treatment. Some other reports also supported the effectiveness of the rituximab against antibody-mediated rejection [10].

\section{Mechanism of ABO blood type related rejection}

In ABO-I organ transplantation, it is believed that early graft failure is caused by a series of responses triggered by antigenantibody reactions between donor blood antigens on the graft and the corresponding antibodies in the recipient's serum [11]. The main reason for a poor result is severe hyperacute rejection due to antidonor $\mathrm{ABO}$ antibodies during the early postoperative period. Once anti-blood type antibodies attach to the blood type antigens on the vascular endothelial cells, this causes damage to the endothelial cells. This phenomenon continues to develop with the production of substances such as cytokines, chemotactic factors, and free radicals, and is accompanied by platelet and complement activation, thrombus formation, granulocyte and macrophage migration, and phagocytosis by granulocytes and macrophages. Moreover, it was reported that the other complications of ABO-I LT, such as hepatic necrosis and intrahepatic biliary complications, were closely related to a high perioperative anti-A or anti-B Ab titers [12]. Thus, most of the efforts to improve the outcome of ABO-I liver transplantation have been directed toward the elimination of anti-blood type antibodies. Particularly, the impact of preformed anti-donor $\mathrm{ABO}$ antibodies and the strategy to reduce their titers play key roles in the success of pediatric ABO-I LDLT [13-15].
Apparently the micro-quantities of anti-A and anti-B antibodies within the blood are adsorbed by endothelial cells in hepatic blood vessels, causing a decrease of the serum antibody titer to zero at approximately several days after transplantation. It is also possible that reperfusion injury from the reinitiation of blood flow encourages endothelial cell damage. Thus, in some cases, localized subclinical rejection may develop and then be reversed mainly by fibrinolysis, resulting in graft survival [16]. During this time, there is no remarkable elevation of serum antibody titer as long as the antibodies are adsorbed within the liver. However, from a pathophysiological perspective, in cases of acute rejection antibody mediated rejection is accompanied by cellular rejection, especially within the first three weeks. Graft loss during this period always involves antibody mediated rejection.

Then, why do grafts survive following ABO-Incompatible liver transplantation? If we examine surviving grafts, we see that even though $\mathrm{ABO}$ blood group antigens are present on the vascular endothelial cells within the graft and even though the recipient's blood contains anti-A/anti-B antibodies, no antibody mediated rejection occurs. In the field of kidney transplantation, this conundrum has been researched aggressively. Some patients with $\mathrm{ABO}$-incompatible kidney grafts have been found within 1 year transplantation to be positive for C4d and have detectable titers of antibody without evidence of graft injury. Resistance to antibody-mediated injury has been observed in a number of experimental and human $\mathrm{ABO}$-incompatible or crossmatch positive allografts, a condition termed 'accomodation,' implying that the endothelium has developed resistance to antibody-mediated injury. However, Fidler et al. [17] reported C4d deposition with histologic abnormalities in 15 of 127 protocol biopsies from $32 \mathrm{ABO}$-incompatible allografts, suggesting that accommodation in the presence of antiABO antibodies is not often complete. Further studies are needed to determine whether true accomodation occurs, or whether the presence of such antibodies in the absence of classical histologic changes simply reflects subtle allograft injury over a long time frame [18].

\section{Recent strategies for ABO-I liver transplantation}

The outcome of the ABO-I liver transplantation relies on the handling of anti-ABO antibodies during the perioperative period. Thus, there have been many trials and errors regarding the routine immunosuppressive protocol. In addition to the regimen used for kidneys, a different approach including periodical steroid pulsing and the use of anti-T lymphocyte antibody was tested. However, these methods not only failed to inhibit liver necrosis and intrahepatic bile duct injury, but also increased the risk of serious infections [11]. Success was reported sporadically, but reproducibility was poor. As a result, no gold standard protocol for the ABO-I liver transplantation was established until the end of the 1990's.

\section{Rituximab}

Rituximab has been approved for the treatment of relapsed or refractory B-cell non-Hodgkin's lymphoma, and autoimmune diseases [19]. It was first used in clinical transplantation in 2002 in Japan in a 22 year-old renal transplant recipient [20]. Since then, rituximab has been adopted based on the experience with rituximab in kidney transplant recipients [7]. As for liver transplantation, Monterio et al. reported the first case of rituximab use in 2003 [8].

Rituximab is a monoclonal chimeric human-murine antiCD20 antibody that depletes the B cells by complement-dependent cytotoxicity, drug-induced apoptotic death, and antibody-dependent cellular cytotoxicity. We had good results in children without using 
rituximab before 2000, but we changed the strategy for ABO-I cases after we experienced a fatal case of antibody mediated rejection in 2000. Namely, we first reported the use of rituximab in LDLT recipients in 2005 [9], and described the successful treatment of rejection episodes in a patient who had not responded to other forms of treatment. Some other reports also supported the effectiveness of rituximab against antibody mediated rejection [21]. Recently, Egawa et al. reported the recommended timing and doses of rituximab for ABO-I LDLT recipients (U). Early administration of rituximab had no significant impact on antibody mediated rejection incidence or patient survival according to the Japanese registry, although at many centers rituximab is administered from 7 to 15 days preoperatively [22-24]. Rituximab was approved for resistant B-cell lymphoma at a dose of $375 \mathrm{mg} / \mathrm{m}^{2}$ weekly for 4 weeks. To deplete normal B cells in an ABO-I recipient, a single dose of rituximab is considered enough. In a recent search of the Japanese registry, it was found that half of the patients were given $500 \mathrm{mg} /$ body, a quarter were given $300 \mathrm{mg} /$ body and the rest were given $375 \mathrm{mg} / \mathrm{m}^{2}$. And there was a tendency toward a higher incidence of antibody mediated rejection in patients administered less than $300 \mathrm{mg} /$ body compared with $500 \mathrm{mg} /$ body or $375 \mathrm{mg} / \mathrm{m}^{2}$. Dose reduction of rituximab could derive in an increase of potential adverse effects in patients with end-stage liver disease, the same as in kidney transplantation; thus, more evidence is needed before recommending dose reduction. The incidence of adverse effects of rituximab was 1.6\%; all recipients recovered and underwent LDLT [22]. Rituximab prophylaxis was tolerated by recipients with end-stage liver diseases. The incidence of bacterial infections and CMV disease after LDLT were similar between the nonrituximab and rituximab groups, but the incidence of fungal infection was significantly lower in the rituximab group. Lower amounts of conventional immunosuppressants might be a reason for the lower occurrence of fungal infections. Anyway, the introduction of rituximab significantly improved the outcome of adult ABO-I LDLT.

\section{Local infusion therapy}

In 1998, a Keio University group of researchers developed an anti-rejection regimen administered by portal vein infusion [5]. Their protocol included multiple perioperative plasma exchange, splenectomy, and a systemic triple immunosuppressive regimen consisting of tacrolimus, methylprednisolone, and cyclophosphamide or amathioprine. In addition to these conventional regimens, they introduced portal vein infusion therapy. They considered that local prophylactic therapy might be more effective and favorable than systemic therapy. The target of this therapy is to control local intravascular coagulation in the graft. Prostaglandin $\mathrm{E}_{1}$, gabexate mesylate and methylprednisolone are infused through the catheter. Prostaglandin $\mathrm{E}_{1}$ improves hepatic blood flow and the microcirculation through its vasodilating effects and inhibits platelet/leukocyte adhesion. Gabexate mesylate is a serine protease inhibitor that inhibits thrombin, $\mathrm{Xa}$, and platelet aggregation. Methylprednisolone has a wide spectrum of anti-inflammatory and immunosuppressive effects. One concern was that portal vein thrombosis increased when portal vein infusion was combined with splenectomy, due to thrombocytosis and decreased splenic vein flow. But the Keio group suggested that the catheter should be inserted from middle colic vein, and the tip of the catheter should be placed peripheral to the superior mesenteric vein [11]. This catheter placement is optimal for a safe and effective therapy.

Another local infusion therapy emerged from Kyoto group [6]. The immunosuppressive therapy included multiple perioperative plasmapheresis, splenectomy, and treatment with tacrolimus, methylprednisolone, and cyclophosphamide. The dose and blood level of tacrolimus were the same as in ABO-compatible cases. In addition to these therapies, they administered an intrahepatic arterial infusion with prostaglandin (PG) E1 alone. The catheter was placed through one of the branches of the hepatic artery after anastomosis. This protocol improved the 1-year survival after ABO-I LDLT to $85 \%$, and decreased the frequency of portal vein thrombosis [25]. However, severe bleeding could occur if the catheter dislocates, either spontaneously or during its removal. Retrospective review of the Japanese national registry has shown that graft survival is not significantly different whether hepatic artery or portal vein infusion therapy is used. Both treatments were collectively designed as "local infusion therapy", and this markedly improved the outcome of the ABO-I LDLT. Local infusion therapy contributed to increase the 2-year adult recipient survival rates after ABOI-LDLT in Japan from around 60\% to $80 \%$ since 2005 (Figure 1).

\section{Plasma exchange}

In general, approximately $3000-4000 \mathrm{ml}$ of plasma will be processed during a single plasma exchange session, allowing the removal of 50$60 \%$ of the IgG or IgM fraction [16]. It is thus safe to assume that the anti-A/anti-B antibody removal rate is approximately $50 \%$ for a single plasma exchange session. Type $\mathrm{AB}$ plasma, which contains neither anti-A nor anti-B antibodies, is thus used as the replacement fluid. However, type $A B$ plasma can contain soluble antigens of types $A$ and $\mathrm{B}$, along with antibodies of unknown immunoreactivity that can act as antigens upon transfusion into the recipient and thus result in an elevated antibody titer.

The presence of anti-A and/or -B antibodies in the recipient and the wide expression of these antigens on endothelial cells, as well as on a variety of parenchymal epithelial cells in the graft, are indeed risks for antibody mediated rejection. The impact of anti-donor $\mathrm{ABO}$ antibodies and the strategy to reduce their titers play key roles in the success of this transplantation. In our institute, a recipient whose titer of IgM or IgG is equal to or greater than 16 is subjected to plasma exchange before transplantation. Plasma exchange is performed 3 or more times until the titer of the antibody becomes equal to or less than 8 [14]. If the titer increases in the early postoperative period, most of the cases suffer antibody mediated rejection. Plasma exchange is useful to decrease the titer temporarily, but we can never controls the ABO-I related antibody mediated rejection using only PE.

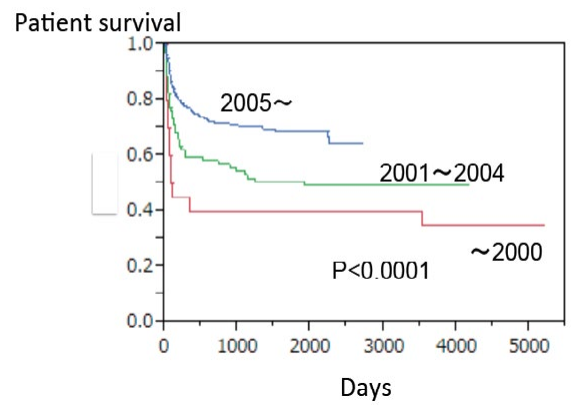

Figure 1. Patient survival curve on ABO-I LDLT patients 16 year-old or older in Japan. The introduction of rituximab and infusion therapy has yielded excellent results after ABO-incompatible LDLT even in adults. Infusion therapy was introduced since 1998, and rituximab was introduced since 2002. Current 2-year patient survival in ABO-compatible LDLT in Japan is around $80 \%$. (Japan Study Group for ABO Blood Type Incompatible Transplantation 2013). 
Table 1. Milestones ABO-I liver transplantation. Improvements of immunosuppressive protocol.

\begin{tabular}{|l|l|l|}
\hline $1970 s^{\prime}$ & Starzl group & ABO-I deceased donor liver transplantation \\
\hline 1991 & Kyoto group & First case of ABO-I living donor liver transplantation in Japan \\
\hline 1998 & Keio group & Portal vein infusion therapy \\
\hline 2001 & Kyoto group & Hepatic artery infusion therapy \\
\hline 2002 & Tohoku group & Rituximab \\
\hline
\end{tabular}

\section{Splenectomy}

The spleen is a major site of antibody production. Thus, splenectomy has been performed in both renal and liver ABO-I transplantations. Some research groups [26-28] reported that splenectomy is needed because of the following reasons: a) splenectomy is expected to eradicate remnant antibody-producing plasma cells that cannot be eliminated by rituximab and plasma exchange; b) splenectomy can significantly reduce excessive portal vein flow, which is critical for small-for-size graft; c) thrombocytopenia or severe splenomegaly affects the quality of life; and d) portal vein infusion therapy with splenectomy and a tripleagent immunosuppression regimen consisting of tacrolimus, steroids, and cyclophosphamide or mycophenolate mofetil is the most effective regime. However, these days, the clinical significance of splenectomy has become a matter of controversy. The spleen represents only around a quarter of the peripheral lymphoid tissue, and splenectomy compromises the immune response because the recipient is deprived of the other immune functions of the spleen and is at an increased risk of infections [25]. The Kyoto group recently reported that there was no statistically significant difference in anti-ABO immunoglobulin $\mathrm{M}$ and immunoglobulin G antibody titers between the "splenectomy" and "nonsplenectomy" groups during the initial 8 weeks [29]. And the clinical outcomes, including antibody mediated rejection, biliary complications, infections, and survival, were similar in both groups. In our experience, the threshold to perform splenectomy is not so high, and its reliability regarding reduction of antibodies is certainly expected, we continue to perform splenctomy so far.

\section{Intravenous immunoglobulin}

Another strategy for ABO-I LDLT is intravenous administration of high-dose IgG (IVIG). The proposed mechanisms include: blockade of Fc receptors on mononuclear phagocytes, direct neutralization of allo-antibodies, inhibition of CD19 expression on activated B-cells, inhibition of complement and inhibition of alloreactive T cells [30]. But in Japan the number of ABO-I LDLT recipients who undergo IVIG therapy is limited. A Kyushu group of researchers reported that three of their four patients with liver cirrhosis received rituximab over 3 weeks before LDLT, followed by PEs and post-LDLT IVIG, resulting in no rebound of isoagglutinin titers. The remaining patient, with fulminant hepatitis, received rituximab 3 days before LDLT, resulting in antibodymediated rejection, successfully treated by IVIG and PE [31].

\section{Other considerations}

\section{Blood adsorption and immunoadsorption}

Biosynsorb $^{\mathrm{R}}$ is an adsorption agent for anti-A and anti-B antibodies in which the determining group, similar to erythrocyte type antigen, is chemically synthesized and bound to silica gel beads. The effects of Biosynsorb $^{\mathrm{R}}$ were demonstrated by Bensinger [32] in 1981. Biosynsorb ${ }^{\mathrm{R}}$ is capable of reducing antibody titer to $1 / 4-1 / 8$ the pretransplant level in a single treatment of 3000-4000 of plasma. In contrast to total plasma exchange, immunoadsorption requires almost no use of exchange fluid.
The cost of the device may be argued.

\section{Histopathology}

Unlike acute cellular or chronic rejection, the diagnosis of antibody mediated rejection in liver allografts is often difficult to establish. However, the early and distinguished histopathological diagnosis of antibody mediated rejection is crucial for the management of ABO-I liver transplantation. The Kyoto group recently reported that among ABO-I liver transplant recipients, complement component 4d (C4d) positivity was not associated with allograft dysfunction or fibrosis [33]. On the contrary, C4d endothelial positivity among ABO compatible recipients was an uncommon event that could be associated with chronic graft damage with or without clinical antibody mediated rejection. We hope for further progress on histopathological diagnosis.

\section{Long-term perspective}

Long-term outcomes of pediatric ABO-I LDLT are not significantly different from ABO compatible cases [34]. Similarly, the survival of living donor kidney series of $\mathrm{ABO}$-incompatible recipients, including adults, in Japan, showed no differences compared with ABO-compatible transplants. This showed that the influence of blood type-related immunological factors was almost eliminated once accommodation is established in the recipient of living donor kidney transplant. In most of the cases, the antibody titers became low in long-surviving kidney transplant recipients [35]. We hope that the same will happen to adult recipients of LT, but it takes more time to show the results. Besides, it might be possible that these advanced immunosuppressive protocols for ABO-I LT are adopted for other organ transplantations in the future.

\section{Conclusion}

Rituximab and local infusion therapy is being used in ABOincompatible LDLT in Japan with good and promising results. The use of rituximab and local infusion therapy are new useful strategies for ABO-incompatible liver transplantation developed in Japan. Similar to those recipients who were subjected to ABO-I LDLT during their childhood without receiving rituximab or infusion therapy and are spending their lives like $\mathrm{ABO}$-compatible or -identical recipients, many adults who received rituximab or infusion therapy are also spending normal daily lives. Finally, in terms of long-term outcome, survival after ABO-incompatible LDLT can be expected to proceed without severe complications if perioperative complications such as antibodymediated rejection are overcome.

\section{References}

1. Kawagishi N, Satomi S (2008) ABO-incompatible living donor liver transplantation new insights into clinical relevance. Transplantation 85: 1523-1525. [Crossref]

2. Alexandre GP, De Bruyere M, Squifflet JP, Moriau M, Latinne D, et al. (1985) Human ABO-incompatible living donor renal homografts. Neth J Med 28: 231-234. [Crossref]

3. Gordon RD, Iwatsuki S, Esquivel CO, Tzakis A, Todo S, et al. (1986) Liver transplantation across ABO blood groups.Surgery 100: 342-348. [Crossref]

4. Gugenheim J, Samuel D, Reynes M, Bismuth H (1990) Liver transplantation across ABO blood group barriers. Lancet 336: 519-523. [Crossref]

5. Tanabe M, Shimazu M, Wakabayashi G, Hoshino K, Kawachi S, et al. (2002) Intraportal infusion therapy as a novel approach to adult $\mathrm{ABO}$-incompatible liver transplantation. Transplantation 73: 1959-1961. [Crossref]

6. Nakamura Y, Matsuno N, Iwamoto H, Yokoyama T, Kuzuoka K, et al. (2004) Successful case of adult ABO-incompatible liver transplantation: beneficial effects of intrahepatic artery infusion therapy: a case report. Transplant Proc 36: 2269-2273. [Crossref]

7. Takahashi K (2007) Recent findings in ABO-incompatible kidney transplantation: classification and therapeutic strategy for acute antibody-mediated rejection due 
to ABO-blood-group-related antigens during the critical period preceding the establishment of accommodation. Clin Exp Nephrol 11: 128-141. [Crossref]

8. Monteiro I, McLoughlin LM, Fisher A, de la Torre AN, Koneru B, et al. (2003) Rituximab with plasmapheresis and splenectomy in abo-incompatible liver transplantation. Transplantation 76: 1648-1649. [Crossref]

9. Usuda M, Fujimori K, Koyamada N, Fukumori T, Sekiguchi S, et al. (2005) Successful use of anti-CD20 monoclonal antibody (rituximab) for ABO-incompatible livingrelated liver transplantation. Transplantation 79: 12-16. [Crossref]

10. Egawa H, Ohmori K, Haga H, Tsuji H, Yurugi K, et al. (2007) B-cell surface marker analysis for improvement of rituximab prophylaxis in ABO-incompatible adult living donor liver transplantation. Liver Transpl 13: 579-588. [Crossref]

11. Tanabe M, Kawachi S, Obara H, Shinoda M, Hibi T, et al. (2010) Current progress in ABO-incompatible liver transplantation.Eur J Clin Invest 40: 943-949. [Crossref]

12. Egawa H, Oike F, Buhler L, Shapiro AM, Minamiguchi S, et al. (2004) Impact of recipient age on outcome of $\mathrm{ABO}$-incompatible living-donor liver transplantation. Transplantation 77: 403-411. [Crossref]

13. Kozaki K, Egawa H, Kasahara M, Oike F, Yoshizawa A, et al. (2005) Therapeutic strategy and the role of apheresis therapy for ABO incompatible living donor liver transplantation.Ther Apher Dial 9: 285-291. [Crossref]

14. Kawagishi N, Takeda I, Miyagi S, Satoh K, Akamatsu Y, et al. (2007) Management of anti-allogeneic antibody elimination by apheresis in living donor liver transplantation. Ther Apher Dial 11: 319-324. [Crossref]

15. Mor E, Skerrett D, Manzarbeitia C, Sheiner PA, Schwartz ME, et al. (1995) Successful use of an enhanced immunosuppressive protocol with plasmapheresis for ABOincompatible mismatched grafts in liver transplant recipients. Transplantation 59: 986990. [Crossref]

16. Takahashi K (2004) Accomodation in ABO-Incompatible kidney transplantation. Amsterdam, Elsevier, 1-209.

17. Fidler ME, Gloor JM, Lager DJ, Larson TS, Griffin MD, et al. (2004) Histologic findings of antibody-mediated rejection in ABO blood-group-incompatible livingdonor kidney transplantation. Am J Transplant 4: 101-107. [Crossref]

18. Sis B, Kaplan B, Halloran PF (2006) Histologic findings from positive crossmatch or ABO-incompatible renal allografts: accomodation or chronic allograft injury? Am $J$ Transplant 6: 1753-1754.[Crossref]

19. Zecca M, De Stefano P, Nobili B, Locatelli F (2001) Anti-CD20 monoclonal antibody for the treatment of severe, immune-mediated, pure red cell aplasia and hemolytic anemia. Blood 97: 3995-3997. [Crossref]

20. Sawada T, Fuchinoue S, Teraoka S (2002) Successful A1-to-O ABO-incompatible kidney transplantation after a preconditioning regimen consisting of anti-CD20 monoclonal antibody infusions, splenectomy, and double-filtration plasmapheresis. Transplantation 74: 1207-1210. [Crossref]

21. Egawa H, Ohmori K, Haga H, Tsuji H, Yurugi K, et al. (2007) B-cell surface marker analysis for improvement of rituximab prophylaxis in ABO-incompatible adult living donor liver transplantation. Liver Transpl 13: 579-588. [Crossref]
22. Egawa H, Teramukai S, Haga H, Tanabe M, Mori A, et al. (2014) Impact of rituximab desensitization on blood-type-incompatible adult living donor liver transplantation: a Japanese multicenter study. Am J Transplant 14: 102-114.[Crossref]

23. Morioka D, Togo S, Kumamoto T, Takeda K, Matsuo K, et al. (2008) Six consecutive cases of successful adult ABO-incompatible living donor liver transplantation: a proposal for grading the severity of antibody-mediated rejection. Transplantation 85: 171-178. [Crossref]

24. Kim BW, Park YK, Kim YB, Wang HJ, Kim MW (2008) Effects and problems of adult $\mathrm{ABO}$-incompatible living donor liver transplantation using protocol of plasma exchange, intra-arterial infusion therapy, and anti-CD20 monoclonal antibody without splenectomy: case reports of initial experiences and results in Korea. Transplant Proc 40: 3772-3777. [Crossref]

25. Raut V, Uemoto S (2011) Management of ABO-incompatible living-donor liver transplantation: past and present trends. Surg Today 41: 317-322. [Crossref]

26. Soejima Y, Muto J, Matono R, Ninomiya M, Ikeda T, et al. (2013) Strategic breakthrough in adult ABO-incompatible living donor liver transplantation: preliminary results of consecutive seven cases. Clin Transplant 27: 227-231. [Crossref]

27. Egawa H, Ozawa K, Takada Y, Teramukai S, Mori A, et al. (2009) Impact of preexisting circulating effector T cells on the outcome of ABO-incompatible adult LDLT. Dig Dis Sci 54: 2253-2263. [Crossref]

28. Egawa H, Teramukai S, Haga H, Tanabe M, Fukushima M, et al. (2008) Present status of ABO-incompatible living donor liver transplantation in Japan. Hepatology 47: 143152. [Crossref]

29. Raut V, Mori A, Kaido T, Ogura Y, Taku I, et al. (2012) Splenectomy does not offer immunological benefits in ABO-incompatible liver transplantation with a preoperative rituximab. Transplantation 93: 99-105. [Crossref]

30. Jordan S, Cunningham-Rundles C, McEwan R (2003) Utility of intravenous immune globulin in kidney transplantation: efficacy, safety, and cost implications. $A m J$ Transplant 3: 653-664. [Crossref]

31. Ikegami T, Taketomi A, Soejima Y, Yoshizumi T, Uchiyama H, et al. (2009) Rituximab, IVIG, and plasma exchange without graft local infusion treatment: a new protocol in ABO incompatible living donor liver transplantation. Transplantation 88: 303-307. [Crossref]

32. Bensinger W (1981) Plasma exchange and immunoadsorption for removal of antibodies prior to ABO incompatible bone marrow transplant. ArtifOrgans 5: 254-258. [Crossref]

33. Salah A, Fujimoto M, Yoshizawa A, Yurugi K, Miyagawa-Hayashino A, et al. (2014) Application of complement component $4 \mathrm{~d}$ immunohistochemistry to ABO-compatible and ABO-incompatible liver transplantation. Liver Transpl 20: 200-209. [Crossref]

34. The Japanese Liver Transplantation Society (2011) Liver transplantation in Japanregistry by the Japanese Liver Transplantation Society-. Isyoku 46: 524-536 (in Japanese with English abstract).

35. Kawagishi N, Takeda I, Miyagi S, Satoh K, Akamatsu Y, et al. (2009) Long-term outcome of ABO-incompatible living-donor liver transplantation: a single-center experience.J Hepatobiliary Pancreat Surg 16: 468-472. [Crossref]

Copyright: $@ 2016$ Kawagishi N. This is an open-access article distributed under the terms of the Creative Commons Attribution License, which permits unrestricted use, distribution, and reproduction in any medium, provided the original author and source are credited. 